\title{
A Case of Orbital Lipoma That Occurred in the Lateral Canthal Region of the Tessier no. 8 Craniofacial Cleft
}

\author{
Nobuhiro Sato*, Tomoaki Kuroki, Yasuyoshi Tosa, Yuki Shimizu, Taro Kusano, \\ Shinya Yoshimoto \\ Division of Plastic and Reconstructive Surgery, Showa University Hospital, Tokyo, Japan \\ Email: hotn310@hotmail.com
}

Received 12 March 2014; revised 10 April 2014; accepted 18 April 2014

Copyright (C) 2014 by authors and Scientific Research Publishing Inc.

This work is licensed under the Creative Commons Attribution International License (CC BY).

http://creativecommons.org/licenses/by/4.0/

c) (i) Open Access

\begin{abstract}
We report a case of a 37-year-old Japanese female. She had a Tessier no. 8 craniofacial cleft and an orbital lipoma in the lateral canthal region. Excisional surgery and pathological examination revealed a genuine lipoma. No other case has been reported in the literature. Here, we present our case of a rare genuine lipoma located in lateral canthal region of the Tessier no. 8 cleft.
\end{abstract}

\section{Keywords}

Lipoma; Tessier no. 8; Craniofacial Cleft

\section{Introduction}

The Tessier no. 8 craniofacial clefts extend from the lateral canthus to the temporal region. A dermatocele might occupy the coloboma of the lateral commissure in some cases. The soft-tissue malformation presents as a true lateral commissure coloboma with absence of the lateral canthus. The bony cleft occurs at the frontozygomatic suture. Tessier noted a notch in this region in patients with Goldenhar's syndrome [1]. The number 8 cleft rarely occurs alone, but is usually associated with other craniofacial clefts (e.g., Goldenhar's syndrome, Treacher-Collins syndrome). There are several reports about orbital tumors associated with the Tessier no. 8 craniofacial cleft (Figure 1(a)).

In most cases, the tumors are dermoid or dermolipomas [2]. We report a case of a 37-year-old Japanese female who had a Tessier no. 8 craniofacial cleft and an orbital lipoma in the lateral canthal region. Excisional surgery was carried out, and pathological examination of the tumor revealed a genuine lipoma. We experienced

${ }^{*}$ Corresponding author.

How to cite this paper: Sato, N., Kuroki, T., Tosa, Y., Shimizu, Y., Kusano, T. and Yoshimoto, S. (2014) A Case of Orbital Lipoma That Occurred in the Lateral Canthal Region of the Tessier no. 8 Craniofacial Cleft. Modern Plastic Surgery, $4,32-34$. http://dx.doi.org/10.4236/mps.2014.42007 
a patient with a rare lipoma located in the lateral canthal region of the Tessier no. 8 cleft.

\section{Method and Result}

A 37-year-old Japanese female presented with a chief complaint of a subcutaneous nodule in the right lateral canthus. On examination of the right lateral canthus, a small nodule was palpable, She had no pain or problems with visual acuity, ocular movement, or palpebral movement, and no visual disturbance. The patient felt that the size of the tumor had gradually increased. There were no features of oculofacial syndrome or other malformations. The Subcutaneous nodule was $1 \mathrm{~cm}$ in diameter in the right canthus, and the right lateral canthus was obliterated by skin separation between the upper and lower eyelids. Palpation revealed no bony fault in the lateral wall of the orbit, and the frontozygomatic suture was normal. In the lateral canthus area, the depth of the conjunctival fornix was obliterated. The contour of the opposite eye was normal. The patient had a lateral interposition of a skin strip between the upper and lower eyelids that disrupted the lateral canthus. We diagnosed the tumor as a Tessier no. 8 cleft tumor. Under local anesthesia, a transverse incision was made on the tumor. The tumor was excised, and skin closure was carried out. Reconstruction of the lateral coloboma was not performed, because the patient rejected it. The excised tissue consisted of yellowish adipose tissue without capsules. It did not extend to the orbital fat. The tumor was located above the orbicularis oculi muscle. The size of the excised tumor was $8 \times 9 \times 5 \mathrm{~mm}$. Tumor tissue sections were stained with hematoxylin and eosin for histopathologic examination. The tumor had no fibrous capsule and consisted of well-differentiated adipose tissue with connective tissue dividing it into small lobules. The final diagnosis confirmed the tumor as a lipoma. There has been no recurrence (Figure $1(\mathbf{b})$ ).

\section{Discussion}

Coloboma of Tessier's no. 8 facial cleft is characterized by a lateral interposition of a skin strip between the upper and lower eyelids that disrupts the continuity of the orbicularis muscle and obliterates the continuity of the depth of the conjunctival fornix in this area [1]-[3]. Orbital lipoma is difficult to distinguish histologically from normal adipose tissue, unless it is well encapsulated. Herniated orbital fat is itself relatively rare. The fat is freely movable and indents easily, and can be repositioned into the orbit, although it may be more prominent by retropulsion of the globe [4]. In the present case, the excised tumor was not encapsulated, but we observed intraoperatively that there was no continuation of the tumor to the orbital fat and the tumor was non-movable. Thus, the excised mass was differentiated from herniated orbital fat and the tumor was diagnosed as a primary orbital lipoma (Figure 2). As our patient had a congenital lateral interposition of a strip of skin between the upper and
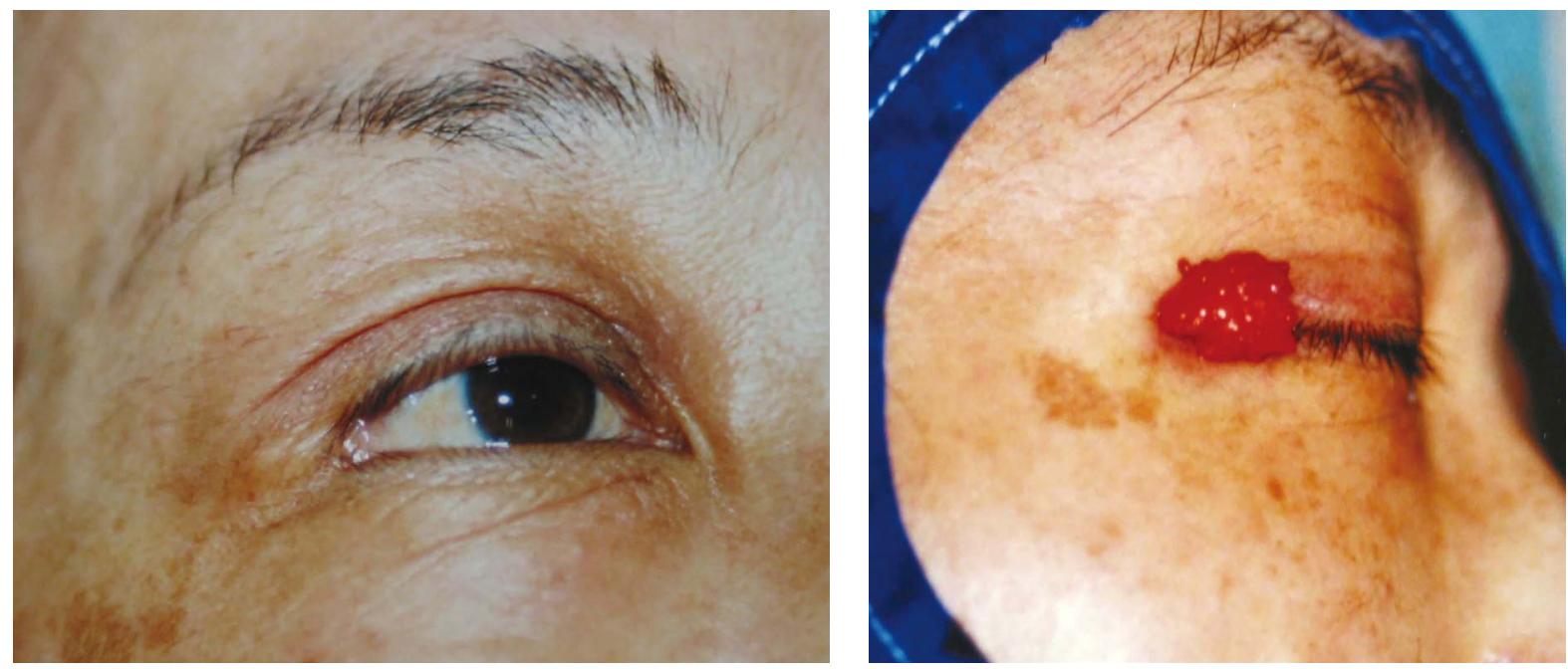

Figure 1. (a) Preoperative appearance, the no. 8 cleft is present in the lateral canthus of the eyelids. Occupying the cleft is a strip of skin. The normal contour of the angle is disrupted; (b) Perioperative findings, the excised tumor was not encapsulated, and it did not continue to the orbital fat. 


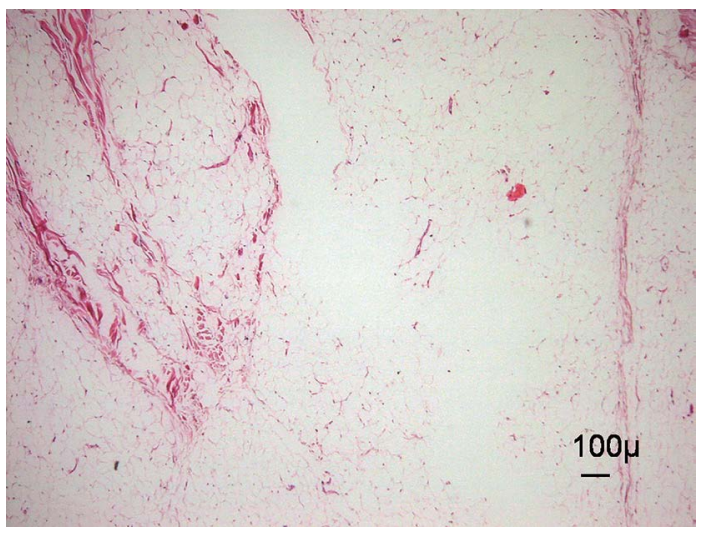

Figure 2. Histological examination, the excised tissue is composed of lobules of mature adipose tissue. Histologically, the tumor was confirmed as a lipoma (hematoxylin-eosin).

lower eyelids disrupting the lateral canthus, we diagnosed the tumor a no. 8 cleft tumor. It is seldom seen as an isolated malformation [1]. There have been some reports of patients with a no. 8 cleft accompanied by dermoids or dermolipomas in the lateral canthus, but not accompanied by an orbital lipoma. There has been no previously published report of the coexistence of a Tessier no. 8 cleft with an orbital lipoma.

Dermolipomas consist of adipose tissue, bundles of dense collagen, and hair follicles covered with keratinized and nonkeratinized stratified squamous epithelium [5]. In the present case, the tumor excised from above the orbicularis oculi muscle consisted of mature adipose tissue, without evidence of dermal appendages, and thus was diagnosed as a lipoma. Lipomas often occur with clefts caused by developmental disorders. As a typical example, in spina bifida occulta, caused by a neural tube defect, lumbosacral lipoma is considered to be the most common malformation leading to this disease. The spinal lipoma penetrates the end of the spinal cord, and continues to the subcutaneous lipoma. Moreover, median cleft face syndrome is often accompanied with dermoids in the orbital region, and also sometimes contains a lipoma of the corpus callosum. Nasopalpebral lipoma-coloboma syndrome is characterized by congenital symmetrical nasopalpebral lipomas, and bilateral symmetrical upper and lower eyelid colobomas. As mentioned above, lipomas often occur with clefts resulting from developmental disorders. Our patient had a Tessier no. 8 cleft with a lipoma in the lateral canthal area and these two conditions might have coexisted by chance, although it is possible that the lipoma was associated with the occurrence of the cleft.

\section{References}

[1] Stephen, J. and Mathes, M.D., Eds. (2006) Plastic Surgery: Embryology, Classifications, and Descriptions of Craniofacial Clefts. 2nd Edition, Saunders Company, Philadelphia, 33-35.

[2] Antonio Fuente-del Campo (1990) Surgical Correction of Tessier Number 8 Cleft. Plastic and Reconstructive Surgery, 86, 658-663. http://dx.doi.org/10.1097/00006534-199010000-00006

[3] Tessier, P. (1976) Anatomical Classification of Facial, Craniofacial, and Laterofacial Clefts. Journal of Maxillofacial Surgery, 4, 69-92. http://dx.doi.org/10.1016/S0301-0503(76)80013-6

[4] Jordan, D.R. and Tse, D.T. (1987) Herniated Orbital Fat. Canadian Journal of Ophthalmology, 22, 173-177.

[5] Fry, C.L. and Leone Jr., C.R. (1994) Safe Management of Dermolipomas. Archives of Ophthalmology, 112, 11141116. http://dx.doi.org/10.1001/archopht.1994.01090200122033 